\title{
Beat-to-beat P-wave Variability Increases From Paroxysmal to Persistent Atrial Fibrillation
}

\author{
Rita Laureanti ${ }^{1,2}$, Stef Zeemering ${ }^{3}$, Matthias D Zink ${ }^{4}$, Valentina D Corino ${ }^{1}$, Angelo Auricchio ${ }^{2,5}$, Luca \\ T Mainardi ${ }^{1}$, Ulrich Schotten ${ }^{3}$ \\ ${ }^{1}$ Politecnico di Milano, Milan, Italy \\ ${ }^{2}$ Center for Computational Modeling in Cardiology, Lugano, Switzerland \\ ${ }^{3}$ Cardiovascular Research Institute Maastricht (CARIM), Maastricht, the Netherlands \\ ${ }^{4}$ RWTH University Hospital Aachen, Aachen, Germany \\ ${ }^{5}$ Cardiocentro Ticino, Lugano, Switzerland
}

\begin{abstract}
Atrial fibrillation (AF) is known to worsen over time. Beat-to-beat $P$-wave variability is used to evaluate the risk of developing AF, but it has not been used to monitor arrhythmia progression in a comprehensive model. The aim of this study is to create a method to measure beat-tobeat $P$-wave variability to evaluate AF types.

ECG recordings of 5 minutes were measured on 159 $A F$ patients. The first three principal components (PCs) of the ECG signal were added to the analysis. The temporal beat-to-beat P-wave variability was assessed through the normalized Euclidean Distance and the Similarity Index. The spatial P-wave similarity was measured as the percentage of variance explained by the first 2 PCs. A binomial logistic regression model was built for each lead and parameter, with AF type as dependent variable. To assess variability due exclusively to the P-waves, we considered, as confounding factors, other sources of ECG-variability, such as the noise level, the variability of the $R R$ series and of the heart axis.

Both temporal (e.g. 0.94 \pm 0.12 for paroxysmal $A F$ and $0.85 \pm 0.28$ for persistent $A F$ in lead I, $p=0.001$ ) and spatial $P$-wave similarities $(95.35 \pm 3.29 \%$ for paroxysmal $A F$ vs $94.44 \pm 4.14 \%$ for persistent $A F, p=0.001$ ) were significantly higher in paroxysmal than in persistent $A F$, suggesting them as promising tools to evaluate AF types.
\end{abstract}

\section{Introduction}

Atrial Fibrillation (AF) is the most common arrhythmia with an important contribution to population morbidity and mortality [1]. Since the onset of the pathology, AF causes electrophysiological and/or structural remodeling in the atria, promoting its perpetuation [1], [2] and a more difficult restoration of sinus rhythm (SR). According to the temporal pattern of the arrhythmia, three types of AF were identified [3]: paroxysmal, when episodes terminate spontaneously, usually within seven days and mostly in less than 24 hours; persistent, when the arrhythmia continues for more than a week, requiring pharmacological or electrical treatment for termination; permanent, when AF last for more than 1 year and cardioversion is not indicated, has failed or has not been attempted. Early knowledge of the AF type can help clinicians in the choice of best treatment and in the application of risk management procedure to avoid adverse events. Beat-to-beat $\mathrm{P}$ wave variability has been associated with higher risk of developing AF [4]-[6] and its increase has been related to the presence of heterogeneous conduction [7].

The aim of this study is to find whether spatiotemporal $\mathrm{P}$-wave variability of AF patients is able to give information about the condition of the atria and discriminate between AF types.

\section{Methods}

\subsection{Population}

The studied population comprised of 159 AF patients enrolled in the Atrial Fibrillation Ablation (AFAB) study, scheduled for AF ablation at the Maastricht University Medical Center (MUMC+). The study protocol was approved by the institutional review board and informed written consent was obtained from each participant. Each subject had a 5-minute ECG recorded with CAM-USB (GE Healthcare, sample frequency $=500 \mathrm{~Hz}$ ) or YRS-100 ECG device (YourRhythmics B.V., sample frequency = $2000 \mathrm{~Hz}$ ). Only subjects presenting with sinus rhythm (SR) were considered in the analysis.

Three additional unipolar leads were added to the standard 12 leads for their sensitivity to high P-wave complexity [8], [9]: A1 (cranial end of sternum, beneath jugulum), A2 (8 cm above V6, mid-axillary line) and A3 (same height as A2, above V9, $4 \mathrm{~cm}$ medial of posterior axillary line). The population characteristics are depicted in Table 1. 
Table 1. Population characteristics.

\begin{tabular}{|c|c|c|c|}
\hline & $\begin{array}{c}\text { Total } \\
\substack{\text { MediantIQR/ } \\
(\mathrm{N})} \\
\end{array}$ & $\begin{array}{l}\text { paroxysmal } \\
\text { Median } \pm \mathrm{QR} / \%(\mathrm{~N})\end{array}$ & $\begin{array}{l}\text { persistent } \\
\text { MediantIQR /\% } \\
\text { (N) }\end{array}$ \\
\hline $\mathrm{N}$ & 159 & 119 & 40 \\
\hline Male & $64 \%(101)$ & $61 \%(73)$ & $70 \%(28)$ \\
\hline Age & $64.2 \pm 12.7$ & $63.7 \pm 12.8$ & $65.4 \pm 13.4$ \\
\hline Height & $176 \pm 16$ & $176 \pm 16$ & $178 \pm 15$ \\
\hline Weight & $84 \pm 19$ & $83 \pm 19$ & $86 \pm 21.8$ \\
\hline BMI & $26.7 \pm 5.5$ & $26.6 \pm 5$ & $27.8 \pm 6.9$ \\
\hline $\mathrm{CHF}^{*}$ & $9 \%(14)$ & $5 \%(6)$ & $20 \%(8)$ \\
\hline Hypertension & $50 \%(80)$ & $47 \%(56)$ & $60 \%(24)$ \\
\hline Diabetes & $8 \%(13)$ & $8 \%(9)$ & $10 \%(4)$ \\
\hline \multicolumn{4}{|c|}{ Echocardiographic findings: } \\
\hline LVEF* & $58 \pm 6$ & $59 \pm 5$ & $58 \pm 8$ \\
\hline LAD & $42 \pm 7$ & $42 \pm 7$ & $42 \pm 7$ \\
\hline $\mathrm{LAV}^{*}$ & $83 \pm 37$ & $80 \pm 29$ & $99 \pm 37$ \\
\hline $\mathrm{RAV}^{*}$ & $54 \pm 32$ & $51 \pm 21$ & $71 \pm 33$ \\
\hline \multicolumn{4}{|c|}{ AF Baseline characteristics: } \\
\hline AF known for: & $60 \pm 72$ & $54 \pm 72$ & $60 \pm 96$ \\
\hline Antiarr & $55 \%(88)$ & $55 \%(65)$ & $58 \%(23)$ \\
\hline
\end{tabular}

\subsection{ECG preprocessing}

The recordings were filtered (high-pass filter, Butterworth, 4th-order, $\mathrm{fc}=0.5 \mathrm{~Hz}$; low-pass filter, FIR, Hamming window, $\mathrm{fc}=80 \mathrm{~Hz}$ and non-linear notch filter $\mathrm{fc}=50 \mathrm{~Hz}$ ) in order to reduce the noise influence on the variability estimate [10]. Then, the first three principal components (PCs) of the ECG leads were added to the analysis.

The Principal Component Analysis (PCA) is a statistical procedure to obtain a set of uncorrelated and orthogonal variables from a dataset. The first component is built to explain the majority of the variance in the data. All the other components are built in an iterative procedure to explain the majority of the remaining variance with the constraint to be orthogonal to all the precedent ones [11]. In this way, the first three components of the ECG signal represent the three more representative directions of heart depolarization.

In order to avoid missing detection of low-amplitude peaks, R-peak detection was performed on the 2 leads with highest QRS amplitude. A window going from 80 ms before and $200 \mathrm{~ms}$ after each R peak was selected. The abnormal beats were identified through the crosscorrelation with an average template and discarded from further analysis [12].

\subsection{P-wave variability analysis}

A similar procedure was applied on the P-waves on lead II (where the atrial activity is more visible and positive): all $\mathrm{P}$ waves were cross-correlated and the 20 most correlated ones were averaged to compute a template. Then, all P-waves were aligned according to the lag at which the cross-correlation function with the template was maximal [10], [12]. Abnormal and false Pwaves were discarded using a clustering procedure on each lead: the PCA was applied on all P-windows and the coefficients of the first 2 PCs were used to characterize each P-window on a 2-D plane. Then, the Density-based spatial clustering of applications with noise (DBSCAN) was applied to cluster the P-windows. In Figure 1 an example is depicted: among the P-windows (top left panels) some false P-waves are present (T-waves). The clustering procedure (top right panel) correctly separates the $\mathrm{T}$-waves from the $\mathrm{P}$-waves, while allowing for the intrinsic variability of the P-waves that is to be assessed (bottom left panel).

The beat-to-beat temporal variability was evaluated on each pair of consecutive beats through the normalized Euclidean Distance (ED) [5], computed as:

$$
E D=\frac{\sqrt{\sum_{k=1}^{K}\left(P_{n+1}[k]-P_{n}[k]\right)^{2}}}{\sqrt{\sum_{k=1}^{K}\left(P_{n+1}[k]\right)^{2}}}, n=1,2, \ldots, N-1
$$

where $\mathrm{P}_{n}$ is the $\mathrm{n}$-th $\mathrm{P}$-wave and $\mathrm{N}$ the total number of $\mathrm{P}$ waves in the recordings. Furthermore, a similarity index (SI) was computed as the cosine of the angle between two consecutive P-waves [10].

This procedure was repeated for each lead and principal component. A median value for each variability metric was computed. In order to evaluate the spatial Pwave similarity, for each beat the PCA was applied on the 15 P-waves (one for each lead) and the percentage of variance explained by the first 2 components computed as a measure of spatial similarity. The median value for all the P-waves was computed [10].

Finally, other sources of variability in the ECG were assessed: the Root Mean Square of Successive Difference (RMSSD) of the RR series (a measure of heart rate variability), the RMSSD of the heart axis and a measure of the noise level in each lead and overall. The noise level was measured as the median value of the standard deviation of each isoelectric segment of $50 \mathrm{~ms}$ preceding a P-wave.

\subsection{Statistical Analysis}

Comparison of clinical characteristics and ECG parameters was performed through Wilcoxon rank sum test. Categorical variables were tested with the twoproportion z-test and reported in number and percentage. A binomial logistic regression model was built for each lead and parameter, with AF type as dependent variable. In order to consider the effect of the variability due only 
to the P-wave, all the models were adjusted for all other sources of ECG-related variability. A p-value $<0.05$ was considered statistically significant. All analyses were performed using Matlab R2017a (The MathWorks, Natick, MA).
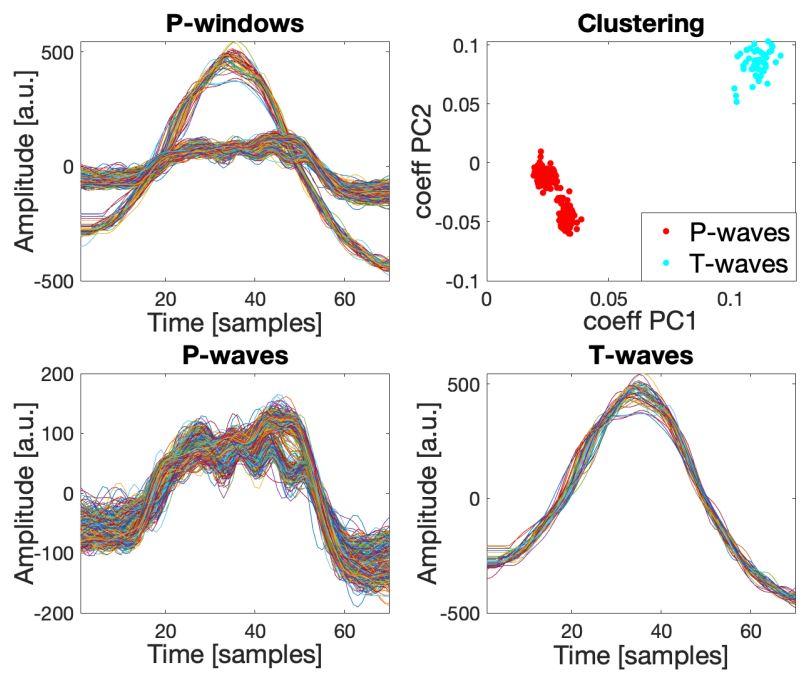

Figure 1: In the top left panel all $\mathrm{P}$-windows (P-waves and false P-waves) are overlapped. In the top right panel each $\mathrm{P}$-window is depicted as a point having as coordinates the coefficients of the first 2 PCs; two major clusters are recognized by DBSCAN, one for the P-waves and one for the T-waves. In the bottom panels the two clusters are showed separately.

\section{Results}

It was found that the ED was consistently higher in persistent AF patients than in paroxysmal AF and, accordingly, SI was lower in persistent AF. In Table 2 the leads that showed a significant difference between paroxysmal and persistent AF are reported for both ED and SI. The spatial similarity was higher in the population with paroxysmal AF $(95.35 \pm 3.29 \%$ for paroxysmal AF vs $94.44 \pm 4.14 \%$ for persistent $\mathrm{AF}, \mathrm{p}=0.001$ ).

\section{Discussion}

To our knowledge, few studies are present in literature about differences between paroxysmal and persistent AF in ECG parameters during SR [10], [13], [14], and none of these focus on beat-to-beat P-wave variability, taking into account also other possible sources of variability in ECG.

During the ECG analysis, an accurate procedure to detect only physiological P-waves was performed, avoiding influence of noisy or false P-waves that could deeply compromise the beat-to-beat comparison. Furthermore, the quantification of other sources of ECGrelated variability allows the adjustment for their effect on the assessment of the association between P-wave variability and AF types.

The beat-to-beat P-wave variability is a direct measure of the changes in the atrial depolarization pathways occurring beat-to-beat. A higher P-wave variability has been associated to higher risk of developing AF in several studies [4]-[6]. Pezzuto et al. showed in an in-silico study

\begin{tabular}{|c|c|c|c|c|c|}
\hline & & $\begin{array}{c}\text { Total } \\
\text { Median } \pm \text { IQR } / \%(\mathbb{N})\end{array}$ & $\begin{array}{l}\text { paroxysmal } \\
\text { Median } \pm I Q R / \%(N)\end{array}$ & $\underset{\text { Median } \pm I Q R / \%(\mathbb{N})}{\text { persistent }}$ & $P=$ \\
\hline$N$ & & 159 & 119 & 40 & \\
\hline \multicolumn{6}{|l|}{ Euclidean } \\
\hline \multirow[t]{7}{*}{ Distance: } & A1 & $(3.56 \pm 2.58) \times 10^{-1}$ & $(3.31 \pm 2.27) \times 10^{-1}$ & $(4.55 \pm 3.31) \times 10^{-1}$ & 0.015 \\
\hline & A2 & $(3.94 \pm 2.49) \times 10^{-1}$ & $(3.61 \pm 2.32) \times 10^{-1}$ & $(4.76 \pm 4.01) \times 10^{-1}$ & 0.001 \\
\hline & I & $(3.98 \pm 3.74) \times 10^{-1}$ & $(3.78 \pm 3.03) \times 10^{-1}$ & $(5.47 \pm 5.10) \times 10^{-1}$ & 0.001 \\
\hline & II & $(2.66 \pm 1.41) \times 10^{-1}$ & $(2.61 \pm 1.14) \times 10^{-1}$ & $(3.03 \pm 2.02) \times 10^{-1}$ & 0.009 \\
\hline & V6 & $(3.8 .2 \pm 1.91) \times 10^{-1}$ & $(3.54 \pm 1.66) \times 10^{-1}$ & $(4.19 \pm 2.76) \times 10^{-1}$ & 0.002 \\
\hline & aVR & $(2.79 \pm 1.80) \times 10^{-1}$ & $(2.66 \pm 1.58) \times 10^{-1}$ & $(3.37 \pm 2.68) \times 10^{-1}$ & 0.001 \\
\hline & $\mathrm{PC} 1$ & $(1.99 \pm 1.29) \times 10^{-1}$ & $(1.92 \pm 1.09) \times 10^{-1}$ & $(2.23 \pm 2.11) \times 10^{-1}$ & 0.009 \\
\hline \multicolumn{6}{|l|}{ Similarity } \\
\hline Index: & A1 & $(9.45 \pm 0.91) \times 10^{-1}$ & $(9.52 \pm 0.79) \times 10^{-1}$ & $(9.13 \pm 1.37) \times 10^{-1}$ & 0.011 \\
\hline & A2 & $(9.30 \pm 1.02) \times 10^{-1}$ & $(9.40 \pm 0.87) \times 10^{-1}$ & $(8.92 \pm 2.01) \times 10^{-1}$ & $<0.001$ \\
\hline & I & $(9.29 \pm 1.58) \times 10^{-1}$ & $(9.37 \pm 1.15) \times 10^{-1}$ & $(8.54 \pm 2.75) \times 10^{-1}$ & 0.001 \\
\hline & II & $(9.69 \pm 0.31) \times 10^{-1}$ & $(9.70 \pm 0.27) \times 10^{-1}$ & $(9.59 \pm 0.61) \times 10^{-1}$ & 0.008 \\
\hline & V6 & $(9.38 \pm 0.56) \times 10^{-1}$ & $(9.45 \pm 0.53) \times 10^{-1}$ & $(9.20 \pm 1.11) \times 10^{-1}$ & 0.001 \\
\hline & aVR & $(9.65 \pm 0.49) \times 10^{-1}$ & $(9.70 \pm 0.43) \times 10^{-1}$ & $(9.51 \pm 0.91) \times 10^{-1}$ & 0.002 \\
\hline & PC1 & $(9.83 \pm 0.25) \times 10^{-1}$ & $(9.84 \pm 0.21) \times 10^{-1}$ & $(9.77 \pm 0.52) \times 10^{-1}$ & 0.001 \\
\hline \multicolumn{2}{|c|}{$\begin{array}{l}\text { Spatial } \\
\text { similarity [\%] }\end{array}$} & $95.06 \pm 3.35$ & $95.35 \pm 3.29$ & $94.44 \pm 4.14$ & 0.001 \\
\hline
\end{tabular}

Table 2. P-wave parameters presenting significant differences between patients with paroxysmal and persistent AF. P-value calculated using a binomial logistic regression model adjusted for other ECG-related variability's sources. 
that the beat-to-beat P-wave variability is related to the degree of heterogeneous atrial conduction and the variability of the sinoatrial node's exit points. According to these findings, a higher P-wave variability in the persistent population was expected, related to the more compromised substrate due to the worsening of the pathology. The results of the study sustain this hypothesis. In a study by Laureanti et al., the beat-to-beat P-wave variability in lead II and in the first principal component was computed to evaluate possible sex-related differences in the Swiss-AF cohort, but also differences between paroxysmal and persistent AF, finding no significant difference related to the AF type [10]. However, a comparison between the AFAB-Registry from Maastricht and the Swiss-AF population shows major demographic and treatment differences. The SwissAF patients were older (on average 6 years), were smaller and lighter, and were more frequently on antiarrhythmic drugs. Furthermore, the clustering procedure implemented in this study to better identify the P-waves to be analyzed was absent in [10] and no source of other ECG-related variability was considered as a confounding variable.

In conclusion, the use of an extended ECG and an accurate procedure in each lead to avoid noisy or false Pwaves that could affect the variability, along with an assessment of other sources of ECG variability that could influence both ED and SI, are useful tools to quantify the degree of atrial remodeling and give preliminary information about the possible stage of the AF.

\section{References}

[1] Y. Iwasaki, K. Nishida, T. Kato, and S. Nattel, "Atrial Fibrillation Pathophysiology," Circulation, vol. 124, 2011.

[2] M. C. E. F. Wijffels, C. J. H. J. Kirchhof, R. Dorland, and M. A. Allessie, "Atrial Fibrillation Begets Atrial Fibrillation: A Study in Awake Chronically Instrumented Goats," Circulation, vol. 92, no. 7, pp. 1954-1968, 1995.

[3] P. Kirchhof et al., "2016 ESC Guidelines for the Management Of Atrial Fibrillation Developed in Collaboration with EACTS," Eur. Heart J., vol. 37, no. 38, pp. 2893-2962, 2016.
[4] V. D. A. Corino et al., "Beat-To-Beat Analysis of P Waves in Patient with Atrial Fibrillation History," in Computing in Cardiology, 2016.

[5] G. Conte et al., "Usefulness of P-Wave Duration and Morphologic Variability to Identify Patients Prone to Paroxysmal Atrial Fibrillation," Am. J. Cardiol., vol. 119, no. 2, pp. 275-279, 2017.

[6] F. Censi et al., "P-Wave Variability and Atrial Fibrillation," Sci. Rep., vol. 6, 2016.

[7] S. Pezzuto et al., "Beat-To-Beat P-Wave Morphological Variability in Patients With Paroxysmal Atrial Fibrillation: An In Silico Study," Europace, vol. 20, no. suppl_3, pp. iii26-iii35, 2018.

[8] T. A. R. Lankveld, S. Zeemering, H. J. G. M. Crijns, and U. Schotten, "The ECG as a Tool to Determine Atrial Fibrillation Complexity," Heart, vol. 100, pp. 1077-1084, 2014.

[9] M. Meo, V. Zarzoso, O. Meste, D. G. Latcu, and N. Saoudi, "Spatial Variability of the 12-Lead Surface ECG as a Tool for Noninvasive Prediction of Catheter Ablation Outcome in Persistent Atrial Fibrillation," IEEE Trans. Biomed. Eng., vol. 60, no. 1, pp. 20-27, 2013.

[10] R. Laureanti et al., "Sex-Related Electrocardiographic Differences in Patients with Different Types of Atrial Fibrillation: Results From the SWISS-AF Study," Int. J. Cardiol., vol. 307, pp. 67-70, 2020.

[11] I. T. Jolliffe, Principal Component Analysis, Second edi. Springer, 2002.

[12] F. Censi et al., "Time-Domain and Morphological Analysis of the P-Wave. Part I: Technical Aspects for Automatic Quantification of P-Wave Features," PACE - Pacing Clin. Electrophysiol., vol. 31, no. 7, pp. 874 883, 2008.

[13] T. Igarashi et al., "Discrimination of Paroxysmal and Persistent Atrial Fibrillation in Patients with NewOnset Atrial Fibrillation," Int. Heart J., 2016.

[14] R. Laureanti et al., "ECG Features to Discriminate between Patients with Paroxysmal and Persistent Atrial Fibrillation," in Congresso del Gruppo Nazionale di Bioingegneria, 2018.

Address for correspondence:

Rita Laureanti

Via Camillo Golgi, 39, 20133 Milano, Italy rita.laureanti@polimi.it 